\title{
The Kinematics Analysis and Modeling about the Constraint Chain of a 3-TPT Parallel Machine Tool
}

\author{
Jianye Guo ${ }^{1, \mathrm{a}}$, Ning Wang ${ }^{1, \mathrm{~b}}$, Xuejun Deng ${ }^{1, \mathrm{c}}$, Liang Zhao ${ }^{2, \mathrm{~d}}$ \\ ${ }^{1}$ Shenyang Aerospace University, Shenyang, Liaoning, China 110136 \\ ${ }^{2}$ Liaoning Institute of Science and Technology, Benxi, Liaoning, China \\ 117022

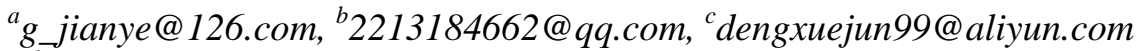 \\ , dzlxh1010@163.com
}

\begin{abstract}
This paper takes a kind of 3-TPT Parallel Machine Tool (PMT) as the objective of research. The positive and inverse kinematics mathematical model of constraint chain are established through the kinematics analysis to the constraint chain of this machine, the inverse kinematics model that has unique solution is also further obtained after the joint angle ranges in the machine were determined. Then the mathematical model about velocity and acceleration of the joints in the constraint chain are also solved in this paper. The analysis results of this paper have laid a theoretical foundation for dynamics analysis, precision analysis, control system design and prototype manufacturing of this Parallel Machine Tool.

Keywords: Parallel Machine Tool (PMT), constraint chain, kinematics analysis, mathematical model

\section{Introduction}

Parallel Machine Tool (PMT) has attracted people's extensive attention from the beginning of its appear. More and more importance has been attached to it in the field of machinery with regard to its research, development and manufacturing [1]. This paper takes a kind of 3-TPT PMT as the objective of research. The kinematics mathematical model of constraint chain have been established through the kinematics analysis to it, thus these have laid the foundation for further study to this PMT.
\end{abstract}




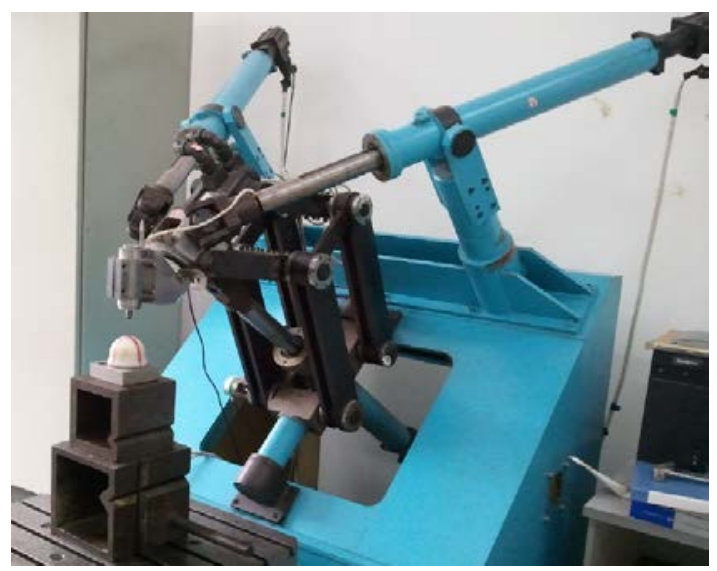

Fig. 1 Structure of 3-TPT PMT

\section{The Structure of Parallel Machine Tool}

The structure of the 3-TPT PMT in this paper is shown in Fig. 1. The parallel mechanism consists of a movable platform, three groups of driving rods, a parallelogram mechanism and a fixed platform. The driving rod groups is the driving chain of the mechanism, which consists of an extensible rod and a swinging rod, and the two rods are connected by moving pair (P). Each driving rod group is joined respectively to the movable platform and the fixed platform through hooker joint $(\mathrm{T})$. The movable platform and the double cross-shaft are connected through the parallelogram mechanism, which is the driven constraint chain of the mechanism. The parallelogram mechanism guarantees that the central axis of the movable platform is always parallel to the axis of rotation of the double cross-shaft. A milling head can be configured on the movable platform, which may rotate around the axial line of the movable platform. After the electric spindle and the tool are being installed on the milling head, machining can be achieved [2,3]. Besides, the vertical-horizontal conversion process can be achieved through milling head rotation.

The fixed platform coordinate system $O-X Y Z$, the movable platform coordinate system $P-x^{\prime} y^{\prime} z^{\prime}$ and the tool coordinate system $D$-xyz for PMT are illustrated in Fig. 2. Take $b_{i x}^{\prime}, b_{i y}^{\prime}, b_{i z}^{\prime}$ as the coordinate value of the joint point of movable platform in the movable platform coordinate system, and $B_{i X}, B_{i Y}, B_{i Z}$ as the coordinate value of the joint point of fixed platform in the fixed platform coordinate system (where $i=1,2,3$ ). The coordinate value of tool tip $D$ in the fixed platform coordinate system is determined as $(x, y, z)$. The tool tip is on the extension line of the movable platform axis. The included angle between the rotary axis $C_{1} C_{2}$ of the double cross-shaft and the horizontal axis is $\pi / 4$ [4]. 


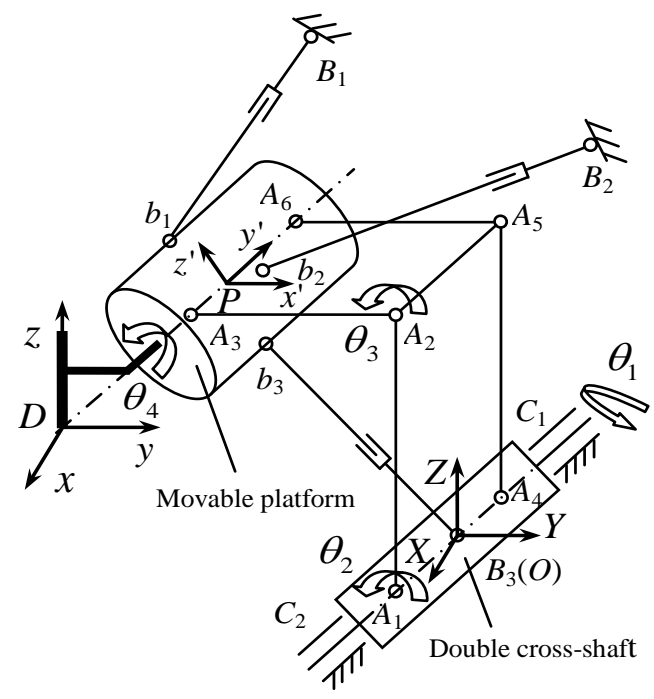

Fig. 2 Sketch of the 3-TPT PMT

\section{The Kinematics Analysis of Constraint Chain}

The task of the kinematics analysis conducted to PMT is to give the kinetic relationship between each components of PMT and to study the relationship and the law of variation for the displacement, velocity and acceleration between all components [5,6]. It is the necessary condition for the overall design of PMT, and the basis of the dynamics analysis, precision analysis and other researches of PMT [7]. Here, the kinematics mathematical model of constraint chain is established by analyzing the kinematics to it.

The Position Analysis of Constraint Chain. The parallelogram mechanism as driven constraint chain has all DOF of 3-TPT parallel mechanism in the structure of PMT and its pairs are all constituted with rotational pair, so it has high rigidity. All coordinate systems are established in the parallelogram mechanism as shown in Fig. 3 and set $A_{1} B_{3}=t_{1}, A_{1} A_{2}=l_{t 1}, A_{2} A_{3}=l_{t 2}, A_{3} P=t_{2}$ [4].

Every coordinate transformation matrix from the fixed platform coordinate system to the tool coordinate system is respectively: 


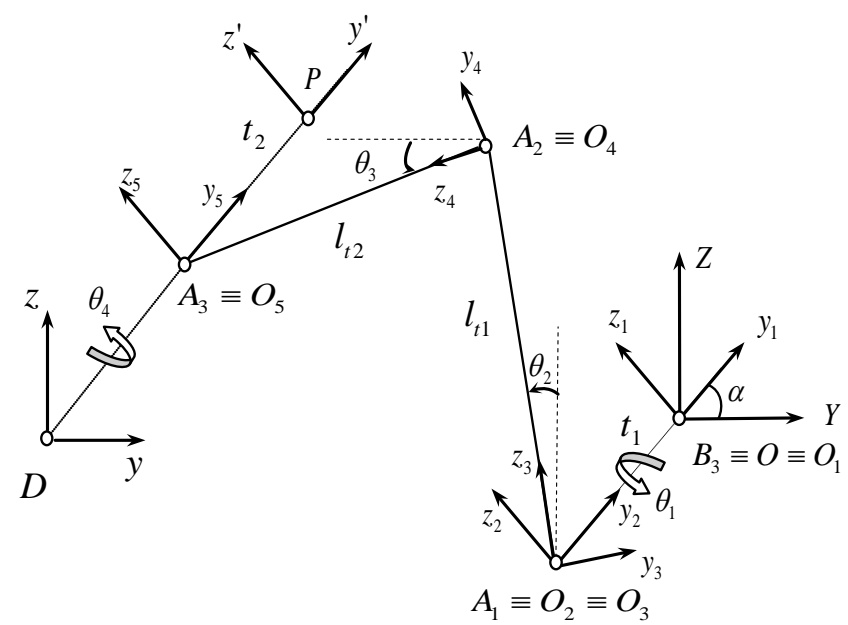

Fig. 3 Sketch of coordinates of constraint chain

$$
\left\{\begin{array}{l}
T_{1}^{O}=\operatorname{Rot}(X, \alpha) \\
T_{2}^{1}=\operatorname{Tran}\left(y_{1},-t_{1}\right) \operatorname{Rot}\left(y_{1}, \theta_{1}\right) \\
T_{3}^{2}=\operatorname{Rot}\left(x_{2},\left(\theta_{2}-\alpha\right)\right) \\
T_{4}^{3}=\operatorname{Tran}\left(z_{3}, l_{t 1}\right) \operatorname{Rot}\left(x_{3},\left(90^{0}-\left(\theta_{2}-\theta_{3}\right)\right)\right) \\
T_{5}^{4}=\operatorname{Tran}\left(z_{4}, l_{t 2}\right) \operatorname{Rot}\left(x_{4},-\left(90^{0}-\left(\alpha-\theta_{3}\right)\right)\right) \\
T_{P}^{5}=\operatorname{Tran}\left(y_{5}, t_{2}\right) \\
T_{D}^{P}=\operatorname{Rot}\left(y^{\prime},-\theta_{1}\right) \operatorname{Rot}\left(x^{\prime},-\alpha\right) \operatorname{Tran}\left(y^{\prime},-l_{d}\right)
\end{array} .\right.
$$

The coordinate transformation matrix between the fixed platform coordinate system and the tool coordinate system can be obtained according to above every adjacent transformation matrix as follow:

$$
T_{D}^{O}=T_{1}^{O} T_{2}^{1} T_{3}^{2} T_{4}^{3} T_{5}^{4} T_{P}^{5} T_{D}^{P} .
$$

Because the direction of every coordinate axis is consistent respectively in the tool coordinate system and fixed platform coordinate system, namely they are translatory relationship mutually, but also the coordinate vector of tool tip is $\vec{r}_{D}=\left(\begin{array}{lll}x & y & z\end{array}\right)^{T}$ in the fixed platform coordinate system, and then may result in:

$$
T_{D}^{O}=\left[\begin{array}{cc}
E & \vec{r}_{D} \\
0 & 1
\end{array}\right] .
$$

Among them, $E$ is a unit matrix. Associating Eq. 2 with Eq. 3, the positive kinematics equation of parallelogram mechanism as constraint chain is obtained as follow: 


$$
\left\{\begin{array}{l}
x=I_{t 1} \cos \left(\alpha-\theta_{2}\right) \sin \theta_{1}+I_{t 2} \sin \left(\alpha-\theta_{3}\right) \sin \theta_{1} \\
y=I_{t 1}\left[\sin \left(\alpha-\theta_{2}\right) \cos \alpha-\cos \left(\alpha-\theta_{2}\right) \cos \theta_{1} \sin \alpha\right] \\
-I_{t 2}\left[\cos \left(\alpha-\theta_{3}\right) \cos \alpha+\sin \left(\alpha-\theta_{3}\right) \cos \theta_{1} \sin \alpha\right]+\left(t_{2}-t_{1}-I_{d}\right) \cos \alpha \\
z=I_{t 1}\left[\sin \left(\alpha-\theta_{2}\right) \sin \alpha+\cos \left(\alpha-\theta_{2}\right) \cos \theta_{1} \cos \alpha\right] \\
-I_{t 2}\left[\cos \left(\alpha-\theta_{3}\right) \sin \alpha-\sin \left(\alpha-\theta_{3}\right) \cos \theta_{1} \cos \alpha\right]+\left(t_{2}-t_{1}-I_{d}\right) \sin \alpha
\end{array}\right.
$$

In the design of the machine structure, because take $\alpha=\pi / 4$, then the inverse kinematics equation of constraint chain is obtained as follow:

$$
\left\{\begin{array}{l}
\theta_{1}=\operatorname{arctg}[\sqrt{2} x /(z-y)] \\
\theta_{2}=\pi / 4-\arcsin \left[\left(b c \pm a \sqrt{a^{2}+b^{2}-c^{2}}\right) /\left(a^{2}+b^{2}\right)\right] \\
\theta_{3}=\pi / 4-\arcsin \left[\left(e f \pm d \sqrt{d^{2}+e^{2}-f^{2}} 0 /\left(d^{2}+e^{2}\right)\right]\right.
\end{array} .\right.
$$

Where,

$$
a=\frac{2 l_{t 1}(z-y)}{\cos \theta_{1}} \quad, \quad b=\sqrt{2}(y+z) l_{t 1}-2\left(t_{2}-t_{1}-l_{d}\right) l_{t 1}
$$

$c=l_{t 1}^{2}-l_{t 2}^{2}+\frac{(z-y)^{2}}{2 \cos ^{2} \theta_{1}}+\frac{1}{2}\left[y+z-\sqrt{2}\left(t_{2}-t_{1}-l_{d}\right)\right]^{2}$,

$$
d=-\sqrt{2}(y+z) l_{t 2}+2\left(t_{2}-t_{1}-l_{d}\right) l_{t 2} \quad, \quad e=\frac{\sqrt{2} l_{t 2}(z-y)}{\cos \theta_{1}},
$$$$
f=-l_{t 1}^{2}+l_{t 2}^{2}+\frac{(z-y)^{2}}{2 \cos ^{2} \theta_{1}}+\frac{1}{2}\left[y+z-\sqrt{2}\left(t_{2}-t_{1}-l_{d}\right)\right]^{2} \text {. }
$$

When the actual joint angles of parallelogram mechanism are limited respectively to the following range: $-\pi / 9<\theta_{1}<\pi / 9,-\pi / 12<\theta_{2}<\pi / 6,-\pi / 4<\theta_{3}<\pi / 6$, then the inverse kinematics equation of parallelogram mechanism that has the unique solution can be obtained as follow:

$$
\left\{\begin{array}{l}
\theta_{1}=\operatorname{arctg}[\sqrt{2} x /(z-y)] \\
\theta_{2}=\pi / 4-\arcsin \left[\left(b c+a \sqrt{a^{2}+b^{2}-c^{2}}\right) /\left(a^{2}+b^{2}\right)\right] \\
\theta_{3}=\pi / 4-\arcsin \left[(z-y) / \sqrt{2} l_{t 2} \cos \theta-l_{t 1} \cos \left(\pi / 4-\theta_{2}\right) / l_{t 2}\right]
\end{array} .\right.
$$

Combining Fig. 2 and Fig. 3, when the PMT is in vertical machining process, the movable platform together with the milling head can be caused an additional rotation because of the $\theta_{1}$ influence. In order to meet the processing requirements that the tool is always perpendicular to the working platform, a rotation $\theta_{4}$ must be input in the milling head to compensate the additional rotation caused by $\theta_{1}$. Also the rotary axis of milling head in the movable platform is always parallel to the rotary axis of double cross-shaft due to the restriction of parallelogram mechanism, so the compensation rotation $\theta_{4}$ and rotation $\theta_{1}$ are the same value, but in the opposite direction.

The Velocity Analysis of Constraint Chain. Take derivation to Eq. 4 on both sides, may result in: 
$\left\{\begin{aligned} \dot{x} & =\dot{\theta}_{1}\left[l_{t 1} \cos \left(\alpha-\theta_{2}\right)+l_{t 2} \sin \left(\alpha-\theta_{3}\right)\right] \cos \theta_{1}+\dot{\theta}_{2} l_{t 1} \sin \left(\alpha-\theta_{2}\right) \sin \theta_{1}-\dot{\theta}_{3} l_{t 2} \cos \left(\alpha-\theta_{3}\right) \sin \theta_{1} \\ \dot{y} & =\dot{\theta}_{1}\left[l_{t 1} \cos \left(\alpha-\theta_{2}\right)+l_{t 2} \sin \left(\alpha-\theta_{3}\right)\right] \sin \theta_{1} \sin \alpha-\dot{\theta}_{2} l_{t 1}\left[\sin \left(\alpha-\theta_{2}\right) \cos \theta_{1} \sin \alpha+\cos \left(\alpha-\theta_{2}\right) \cos \alpha\right] \\ & +\dot{\theta}_{3} l_{t 2}\left[\cos \left(\alpha-\theta_{3}\right) \cos \theta_{1} \sin \alpha-\sin \left(\alpha-\theta_{3}\right) \cos \alpha\right] \\ \dot{z} & =-\dot{\theta}_{1}\left[l_{t 1} \cos \left(\alpha-\theta_{2}\right)+l_{t 2} \sin \left(\alpha-\theta_{3}\right)\right] \sin \theta_{1} \cos \alpha+\dot{\theta}_{2} l_{t 1}\left[\sin \left(\alpha-\theta_{2}\right) \cos \theta_{1} \cos \alpha-\cos \left(\alpha-\theta_{2}\right) \sin \alpha\right] \\ & \quad \dot{\theta}_{3} l_{t 2}\left[\cos \left(\alpha-\theta_{3}\right) \cos \theta_{1} \cos \alpha+\sin \left(\alpha-\theta_{3}\right) \sin \alpha\right]\end{aligned}\right.$

(7)

The above equation is written in matrix form on the style, then the velocity model of the joints in the constraint chain can be obtained [8,9]:

$V=\left[\begin{array}{ccc}\dot{x} & \dot{y} & \dot{z}\end{array}\right]^{\prime}=J_{P} \omega_{\theta}=J_{P}\left[\begin{array}{lll}\dot{\theta}_{1} & \dot{\theta}_{2} & \dot{\theta}_{3}\end{array}\right]^{\prime}$.

Where, $J_{P}$ is Jacobian matrix of constraint chain in the PMT, from Eq. 7 can be obtained:

$J_{p}=\left[\begin{array}{ccc}{\left[I_{t 1} \cos \left(\alpha-\theta_{2}\right)+I_{t 2} \sin \left(\alpha-\theta_{3}\right)\right] \cos \theta_{1}} & I_{t 1} \sin \left(\alpha-\theta_{2}\right) \sin \theta_{1} & -I_{t 2} \cos \left(\alpha-\theta_{3}\right) \sin \theta_{1} \\ {\left[I_{t 1} \cos \left(\alpha-\theta_{2}\right)+I_{t 2} \sin \left(\alpha-\theta_{3}\right)\right] \sin \theta_{1} \sin \alpha} & -I_{t 1}\left[\sin \left(\alpha-\theta_{2}\right) \cos \theta_{1} \sin \alpha+\cos \left(\alpha-\theta_{2}\right) \cos \alpha\right] & I_{t 2}\left[\cos \left(\alpha-\theta_{3}\right) \cos \theta_{1} \sin \alpha-\sin \left(\alpha-\theta_{3}\right) \cos \alpha\right] \\ -\left[l_{t 1} \cos \left(\alpha-\theta_{2}\right)+I_{t 2} \sin \left(\alpha-\theta_{3}\right)\right] \sin \theta_{1} \cos \alpha & I_{t 1}\left[\sin \left(\alpha-\theta_{2}\right) \cos \theta_{1} \cos \alpha-\cos \left(\alpha-\theta_{2}\right) \sin \alpha\right] & -I_{t 2}\left[\cos \left(\alpha-\theta_{3}\right) \cos \theta_{1} \cos \alpha+\sin \left(\alpha-\theta_{3}\right) \sin \alpha\right]\end{array}\right]$

Then $J_{P}^{-1}$ that is inverse matrix to Jacobian matrix $J_{P}$ of constraint chain is:

$J_{P}^{-1}=\left[\begin{array}{ccc}\frac{\cos \theta_{1}}{I_{t 1} \cos \left(\alpha-\theta_{2}\right)+I_{t 2} \sin \left(\alpha-\theta_{3}\right)} & \frac{\sin \theta_{1} \sin \alpha}{I_{t 1} \cos \left(\alpha-\theta_{2}\right)+I_{t 2} \sin \left(\alpha-\theta_{3}\right)} & -\frac{\sin \theta_{1} \cos \alpha}{I_{t 1} \cos \left(\alpha-\theta_{2}\right)+I_{t 2} \sin \left(\alpha-\theta_{3}\right)} \\ \frac{\sin \theta_{1} \sin \left(\alpha-\theta_{3}\right)}{I_{t 1} \cos \left(\theta_{2}-\theta_{3}\right)} & -\frac{\cos \theta_{1} \sin \left(\alpha-\theta_{3}\right) \sin \alpha+\cos \left(\alpha-\theta_{3}\right) \cos \alpha}{l_{t 1} \cos \left(\theta_{2}-\theta_{3}\right)} & \frac{\cos \theta_{1} \sin \left(\alpha-\theta_{3}\right) \cos \alpha-\cos \left(\alpha-\theta_{3}\right) \sin \alpha}{l_{t 1} \cos \left(\theta_{2}-\theta_{3}\right)} \\ -\frac{\sin \theta_{1} \cos \left(\alpha-\theta_{2}\right)}{I_{t 2} \cos \left(\theta_{2}-\theta_{3}\right)} & \frac{\cos \theta_{1} \cos \left(\alpha-\theta_{2}\right) \sin \alpha-\sin \left(\alpha-\theta_{2}\right) \cos \alpha}{I_{t 2} \cos \left(\theta_{2}-\theta_{3}\right)} & -\frac{\cos \theta_{1} \cos \left(\alpha-\theta_{2}\right) \cos \alpha+\sin \left(\alpha-\theta_{2}\right) \sin \alpha}{I_{t 2} \cos \left(\theta_{2}-\theta_{3}\right)}\end{array}\right]$

The Acceleration Analysis of Constraint Chain. Take derivation to Eq. 8 on both sides, may result in:

$$
\left[\begin{array}{lll}
\ddot{x} & \ddot{y} & \ddot{z}
\end{array}\right]^{\prime}=J_{P}\left[\begin{array}{lll}
\ddot{\theta}_{1} & \ddot{\theta}_{2} & \ddot{\theta}_{3}
\end{array}\right]^{\prime}+\frac{d J_{P}}{d t}\left[\begin{array}{lll}
\dot{\theta}_{1} & \dot{\theta}_{2} & \dot{\theta}_{3}
\end{array}\right] .
$$

Then

$$
\left.\left[\begin{array}{lll}
\ddot{\theta}_{1} & \ddot{\theta}_{2} & \ddot{\theta}_{3}
\end{array}\right]^{\prime}=J_{P}^{-1}\left[\begin{array}{lll}
\ddot{x} & \ddot{y} & \ddot{z}
\end{array}\right]^{\prime}-\frac{d J_{P}}{d t}\left[\begin{array}{lll}
\dot{\theta}_{1} & \dot{\theta}_{2} & \dot{\theta}_{3}
\end{array}\right]^{\prime}\right] .
$$

Result can be obtained:

$$
\left.\left[\begin{array}{lll}
\ddot{\theta}_{1} & \ddot{\theta}_{2} & \ddot{\theta}_{3}
\end{array}\right]^{\prime}=J_{P}{ }^{-1}\left[\begin{array}{lll}
\ddot{x} & \ddot{y} & \ddot{z}
\end{array}\right]^{\prime}+J_{P}^{1}\left[\begin{array}{lll}
\dot{\theta}_{1}^{2} & \dot{\theta}_{2}^{2} & \dot{\theta}_{3}^{2}
\end{array}\right]^{\prime}+J_{P}^{2}\left[\begin{array}{lll}
\dot{\theta}_{1} \dot{\theta}_{2} & \dot{\theta}_{2} \dot{\theta}_{3} & \dot{\theta}_{3} \dot{\theta}_{1}
\end{array}\right]^{\prime}\right] \text {. }
$$

Where, $J_{P}^{1}$ and $J_{P}^{2}$ are 


$$
\begin{aligned}
& J_{P}^{1}=\left[\begin{array}{ccc}
{\left[l_{t 1} \cos \left(\alpha-\theta_{2}\right)+l_{t 2} \sin \left(\alpha-\theta_{3}\right)\right] \sin \theta_{1}} & l_{t 1} \cos \left(\alpha-\theta_{2}\right) \sin \theta_{1} & l_{t 2} \sin \left(\alpha-\theta_{3}\right) \sin \theta_{1} \\
-\left[l_{t 1} \cos \left(\alpha-\theta_{2}\right)+l_{t 2} \sin \left(\alpha-\theta_{3}\right)\right] \cos \theta_{1} \sin \alpha & l_{t 1}\left[\sin \left(\alpha-\theta_{2}\right) \cos \alpha+\cos \left(\alpha-\theta_{2}\right) \cos \theta_{1} \sin \alpha\right] & -l_{t 2}\left[\cos \left(\alpha-\theta_{3}\right) \cos \alpha+\sin \left(\alpha-\theta_{3}\right) \cos \theta_{1} \sin \alpha\right] \\
{\left[l_{t 1} \cos \left(\alpha-\theta_{2}\right)+l_{t 2} \sin \left(\alpha-\theta_{3}\right)\right] \cos \theta_{1} \cos \alpha} & l_{t 1}\left[\sin \left(\alpha-\theta_{2}\right) \sin \alpha+\cos \left(\alpha-\theta_{2}\right) \cos \theta_{1} \cos \alpha\right] & -l_{t 2}\left[\cos \left(\alpha-\theta_{3}\right) \sin \alpha-\sin \left(\alpha-\theta_{3}\right) \cos \theta_{1} \cos \alpha\right]
\end{array}\right] \\
& J_{P}^{2}=\left[\begin{array}{ccc}
-2 l_{t 1} \sin \left(\alpha-\theta_{2}\right) \cos \theta_{1} & 0 & 2 l_{t 2} \cos \left(\alpha-\theta_{3}\right) \cos \theta_{1} \\
-2 l_{t 1} \sin \left(\alpha-\theta_{2}\right) \sin \theta_{1} \sin \alpha & 0 & 2 l_{t 2} \cos \left(\alpha-\theta_{3}\right) \sin \theta_{1} \sin \alpha \\
2 l_{t 1} \sin \left(\alpha-\theta_{2}\right) \sin \theta_{1} \cos \alpha & 0 & -2 l_{t 2} \cos \left(\alpha-\theta_{3}\right) \sin \theta_{1} \cos \alpha
\end{array}\right] .
\end{aligned}
$$

\section{Summary}

The kinematics analysis is carried out to the constraint chain of 3-TPT PMT in this paper, the positive and inverse kinematics mathematical model of constraint chain are established, the inverse kinematics model that has unique solution is also obtained based on the joint angle ranges are determined. In addition, the mathematical model about velocity and acceleration of the joints in the constraint chain are also solved in this paper. These have laid a theoretical foundation for dynamics analysis, precision analysis, control system design and prototype manufacturing of this PMT.

\section{References}

[1] Z. Huang, L.F. Kong and Y.F. Fang. The parallel robot mechanism and control theory, China: China Machine Press, 1997, pp:1-268.

[2] X.Z. Meng. Study on a new type of horizontal-vertical conversion three-chain Hybrid Machine Tool's design, Ph.D., Northeastern University, China, 2004, pp:40-75.

[3] Wang J.G, Gosselin C. Static Balancing of Spatial Four-degree-of-freedom Parallel Mechanisms, Mechanism and Machine Theory, 2000, 35(5), pp:563- 592.

[4] J.Y. Guo. Research on the accuracy analysis and relevant problems of lower-mobility tripod hybrid machine tools, Ph.D., Northeastern University, China, 2009, pp:35-43.

[5] L. Woo and F. Freudenstein. Application of line geometry to theoretical kinematics and the kinematic analysis of mechanical systems, Mechanisms, 1970(5), pp:417-460.

[6] H.P. Shen. A new method and automatic generation for kinematic analysis of complex planar linkages based on the ordered single-opened-chains, Proc of ASME Mechanisms Conference, Minneapolis, 1994(70), pp:493-500. 
[7] Z. Huang and Y.F. Fang. Kinematic characteristics analysis of 3-DOF in parallel actuated pyramid mechanisms, Mechanism and Machine Theory, 1996, 31(8), pp:1009-1018.

[8] Y.L. Xiong and H. Ding. Robotics, China: China Machine Press, 1993, pp:4-38.

[9] J.P. Merlet. Parallel robots, Netherland: Kluwer Academic Publishers, 2000, pp:183-232. 